\title{
Evaluation of the cytotoxicity of gold nanoparticle-quercetin complex and its potential as a drug delivery vesicle
}

\author{
Pyo June Pak ${ }^{1}$ Eun Byeol Go ${ }^{1} \cdot$ Min Hee Hwang ${ }^{1}$ Dong Gun Lee ${ }^{1}$ Mi Ju Cho \\ - Yong Hoon Joo ${ }^{1}$ " Namhyun Chung ${ }^{1}$ (D)
}

Received: 26 March 2016 / Accepted: 29 March 2016 / Published Online: 30 June 2016

(C) The Korean Society for Applied Biological Chemistry 2016

\begin{abstract}
Recently, conjugates of medicinal herb-derived bioflavonoids, such as quercetin, and gold nanoparticles (GNPs) have gained attention as targeted drug delivery systems. In the present study, because quercetin is an important flavonoid with anticancer, anti-inflammatory, and anti-oxidant properties, GNP-quercetin complexes (GNPQs) were synthesized to investigate possible adverse effects such as cytotoxicity. We found that while quercetin was cytotoxic, GNPQs were not cytotoxic towards the RAW 264.7 and THP-1 cell lines. Therefore, GNPQs may serve as a potential drug delivery system for cancer treatment.
\end{abstract}

Keywords Citrate synthesis · Drug delivery system · Gold nanoparticle $\cdot$ Quercetin

\section{Introduction}

In the past 10 years, medicinal herbs and their derivatives have gained interest for use as complementary and alternative medicines. Complementary and alternative medicines have been employed for numerous purposes, including disease prevention and health promotion. They have also been used as adjuvants of pharmaceuticals in cancer patients who require long-term medical care (Harris et

Pyo June Pak and Eun Byeol Go contributed equally to this study.

Namhyun Chung $(\bowtie)$

E-mail: nchung@korea.ac.kr

${ }^{1}$ Department of Biosystems Engineering, College of Life Sciences and Biotechnology, Korea University, Seoul 136-713, Republic of Korea

This is an Open Access article distributed under the terms of the Creative Commons Attribution Non-Commercial License (http://creativecommons. org/licenses/by-nc/3.0/) which permits unrestricted non-commercial use, distribution, and reproduction in any medium, provided the original work is properly cited. al. 2012). Therefore, many researchers have focused not only on the discovery of new bioactive molecules from medicinal herbs, but also on their mechanisms of biological activities (Rubio et al. 2013). Studies of diverse medicinal herbs have confirmed the presence of glycosides, saponins, tannins, and flavonoids in plant extracts (Loganayaki et al. 2012; Sidhu and Sharma 2013).

Recently, gold nanoparticles (GNPs) have been extensively studied for their application as targeted drug delivery systems because of their unique physical and chemical properties (Pal et al. 2013). Many forms of GNPs, including spheres, rods, stars, pyramids, and worms, have been employed as drug delivery systems (Kumar et al. 2013). However, before GNPs can be applied for cancer treatment, they must be modified with other molecules, such as polyphenolic compounds, to prevent aggregation (Wang et al. 2007). Therefore, in the present study, we focused on the modification and conjugation of GNPs with quercetin. Quercetin is derived from medicinal herbs and is an important bio-flavonoid with anticancer, anti-inflammatory, and anti-oxidant properties (Russo et al. 2012; Russo et al. 2014). Before further development, the GNP-quercetin complex (GNPQ) must be evaluated for its ability to cause adverse effects, such as cytotoxicity.

\section{Materials and Methods}

First, GNPs and GNPQs were synthesized using the citrate reduction method by reducing $\mathrm{Au}^{+3}$ to $\mathrm{Au}^{0}$ (Verma et al. 2014). Briefly, 20 $\mathrm{mL}$ of $1 \mathrm{mM}$ tetrachloroauric acid (Sigma-Aldrich, St. Louis, MO, USA) was boiled for $5 \mathrm{~min}$ on a pre-heated stir plate. Next, $2 \mathrm{~mL}$ of $1 \%$ trisodium citrate dehydrate (Sigma-Aldrich) was added. This solution was mixed on a stir plate until the solution was turned ruby-red. The solution was removed from the stir plate and stored at $4{ }^{\circ} \mathrm{C}$ until use. The GNPQs were characterized using the Nanoparticle Sizer and Zeta Potential Analyzer (90Plus, Brookhaven Instruments Co., Holtsville, NY, USA). 


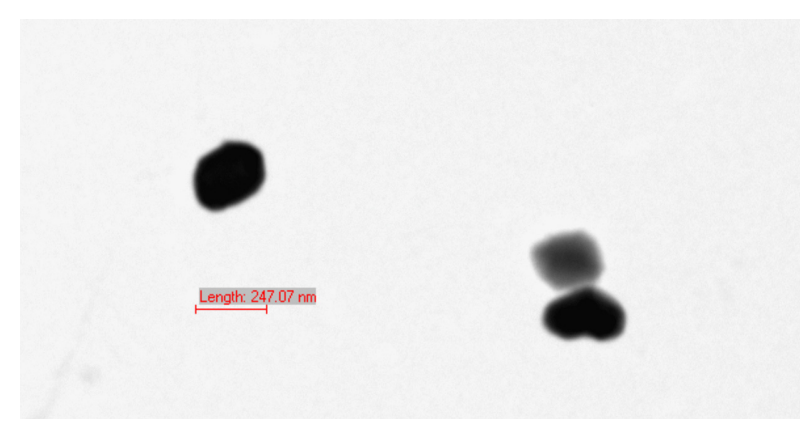

Fig. 1 Observation of gold nanoparticle-quercetin complexes (GNPQs) by transmission electron microscopy

To evaluate the cytotoxicity of the GNPQs, 2 immune cell lines were employed: murine macrophage cell line (Raw 264.7; ATCC No. TIB-71) and human acute monocytic leukemia cell line (THP-1; ATCC No. TIB-202). All cell lines were obtained from American Type Culture Collection (ATCC, Manassas, VA, USA). Raw 264.7 cells were plated at a density of $2.5 \times 10^{5}$ cells $/ \mathrm{mL}$ onto Dulbecco's modified eagle's medium (DMEM; Welgene, Seoul, Korea) supplemented with $10 \%$ heat-inactivated fetal bovine serum (Welgene), $100 \mathrm{U} / \mathrm{mL}$ penicillin, and $100 \mu \mathrm{g} / \mathrm{mL}$ streptomycin. THP-1 cells were plated at a density of $5.0 \times 10^{5}$ cells $/ \mathrm{mL}$ on RPMI 1640 (Welgene) supplemented with $10 \%$ heat-inactivated fetal bovine serum, $0.05 \mathrm{mM}$ 2-mercaptoethanol, $100 \mathrm{U} / \mathrm{mL}$ penicillin, and $100 \mu \mathrm{g} / \mathrm{mL}$ streptomycin. All cells were cultured in a $37^{\circ} \mathrm{C}$ and $5 \% \mathrm{CO}_{2}$ atmosphere. THP-1 cells were differentiated with the addition of phorbol 12-myristate 13-acetate (SigmaAldrich) prior to conducting the experiments (Kim et al. 2015).

The extent of cell viability was measured using the WST-1 assay with the PreMix WST-1 Cell Proliferation Assay System (Takara Bio, Inc., Shiga, Japan) according to the manufacturer's protocol. Absorbance was detected at $490 \mathrm{~nm}$ using a microplate reader (EL800, BioTek, Winooski, VT, USA). The extent of cytotoxicity was measured using the lactate dehydrogenase (LDH) leakage assay with an LDH Cytotoxicity Detection Kit (Takara Bio, Inc.) according to the manufacturer's protocol. Cells treated with quercetin or GNPQs were centrifuged to analyze the extent of LDH release into the supernatant. Absorbance was measured using a microplate reader (EL800, BioTek) at a wavelength of $490 \mathrm{~nm}$.

\section{Results and discussion}

The size of the GNPQs was measured using Nanoparticle Sizer to be $236.9 \pm 16.3 \mathrm{~nm}$ and the zeta potential was $-101.88 \pm 11.72 \mathrm{mV}$. Figure 1 shows a transmission electron microscopic image of the GNPQs synthesized in this experiment (TEM; JEM-1400, Jeol Ltd., Tokyo, Japan). Therefore, GNPQs were stable in solution because the zeta potential value was less than $-40 \mathrm{mV}$ (Jung et al. 2014).

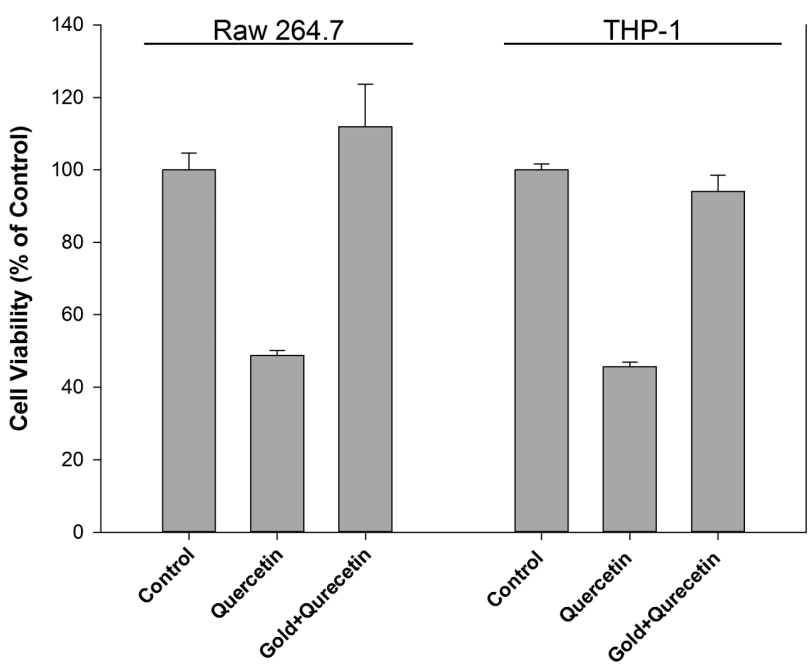

Fig. 2 Cell viability following treatment with quercetin or the gold nanoparticle-quercetin complex (GNPQ) in Raw 264.7 and THP-1 cell lines. Measurement of cell viability was examined using the WST-1 assay. Cell viability was expressed as a percentage of the control. Data were reported as the mean \pm standard deviation of 3 experiments

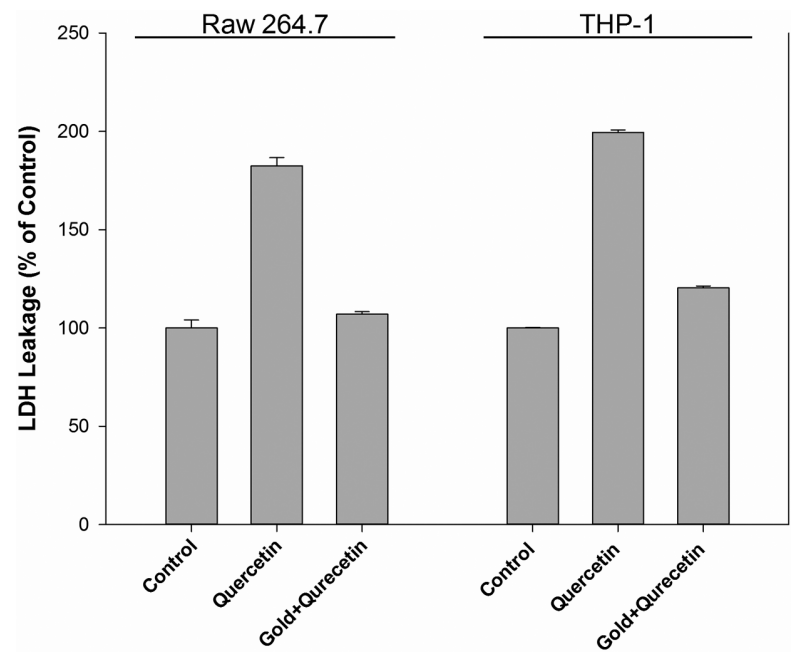

Fig. 3 Cytotoxicity following treatment with quercetin or the gold nanoparticle-quercetin complex (GNPQ) in Raw 264.7 and THP-1 cell lines. Cytotoxicity was measured using the lactate dehydrogenase (LDH) leakage assay. The extent of LDH leakage was expressed as a percentage of the control. Data were reported as the mean \pm standard deviation of 3 experiments

The rate of cell viability in the presence of GNPQs was 111.9 $\pm 11.6 \%$ for Raw 264.7 cells and $94.0 \pm 4.5 \%$ for THP- 1 cells (Fig. 2). The rate of cell viability in the presence of quercetin was $48.8 \pm 1.3 \%$ for Raw 264.7 cells and $45.7 \pm 1.2 \%$ for THP- 1 cells. Thus, quercetin was more cytotoxic than the GNPQs. For this experiment, we employed an $\mathrm{IC}_{50}$ value of $50 \mu \mathrm{M}$ for quercetin which was determined with various cell lines including PANC-1, A549, and MCF-7 (data not shown). The results showed that the GNPQs were less cytotoxic than quercetin, suggesting that the 
degree of cytotoxicity for quercetin was decreased by conjugation of quercetin to the GNPs. The WST-1 assay measures the change in the extent of tetrazolium salt cleavage to formazan by cellular mitochondrial dehydrogenase in living cells (Jung et al. 2014; Kim et al. 2015). Our results indicate that cellular metabolism was more active in the presence of GNPQs than in the presence of quercetin.

The extent of LDH leakage following GNPQ treatment was $107.1 \pm 1.2 \%$ for Raw 264.7 cells and $120.4 \pm 0.9 \%$ for THP-1 cells (Fig. 3). The values were not significantly different from the control. However, the extent of LDH leakage following quercetin treatment significantly increased, with values of $182.5 \pm 4.1 \%$ for Raw 264.7 cells and $199.5 \pm 1.2 \%$ for THP- 1 cells. These results also showed that the GNPQs were less cytotoxic than quercetin and confirmed that the degree of cytotoxicity for quercetin decreased upon conjugation with GNPs.

Quercetin has been shown in previous studies to have anticancer and anti-oxidant properties (Russo et al. 2012; Russo et al. 2014). In the present study, the cytotoxicity of quercetin was observed in 2 different immune cell lines. However, when quercetin was conjugated to GNPs, no cytotoxicity was observed. Pal et al. (2013) have shown that the coating or conjugation of GNPs with other molecules is required for the use of GNPs as drug delivery vesicles. The present results demonstrate that GNPQs may be useful as drug delivery vesicles because GNPQs were not cytotoxic. Thus, GNPQs may be used as a drug delivery system in cancer treatment, but further studies are required to determine the detailed mechanisms that GNPQs target biomolecules such as transferrin receptors, integrin, epidermal growth factor receptor, and bombesin receptors (Pal et al. 2013). Our results provide a foundation for the application of such targeted drug delivery systems in cancer therapy.

Acknowledgments This work was supported by a grant from National Research Foundation of Korea. (Grant No. NRF-2015R1A2A2A0100650).

\section{References}

Harris PE, Cooper KL, Relton C, Thomas KJ (2012) Prevalence of complementary and alternative medicine (CAM) use by the general population: a systematic review and update. Int J Clin Pract 66: 924-939

Jung HJ, Pak PJ, Park SH, Ju JE, Kim JS, Lee HS, Chung N (2014) Silver wire amplifies the signaling mechanism for IL-1beta production more than silver submicroparticles in human monocytic THP-1 cells. PLoS One 9: e112256

Kim BI, Joo YH, Pak PJ, Kim JS, Chung N (2015) Different shapes of $\mathrm{Al}_{2} \mathrm{O}_{3}$ particles induce differential cytotoxicity via a mechanism involving lysosomal destabilization and reactive oxygen species generation. J Korean Soc Appl Biol Chem 58: 433-442

Kumar A, Zhang X, Liang XJ (2013) Gold nanoparticles: emerging paradigm for targeted drug delivery system. Biotechnol Adv 31: 593-606

Loganayaki N, Siddhuraju P, Manian S (2012) Antioxidant, anti-inflammatory and anti-nociceptive effects of Ammannia baccifera L. (Lythracceae), a folklore medicinal plant. J Ethnopharmacol 140: 230-233

Pal R, Panigrahi S, Bhattacharyya D, Chakraborti AS (2013) Characterization of citrate capped gold nanoparticle-quercetin complex: Experimental and quantum chemical approach. J Mol Struct 1046: 153-163

Rubio L, Motilva MJ, Romero MP (2013) Recent advances in biologically active compounds in herbs and spices: a review of the most effective antioxidant and anti-inflammatory active principles. Crit Rev Food Sci Nutr 53: 943-953

Russo GL, Russo M, Spagnuolo C, Tedesco I, Bilotto S, Iannitti R, Palumbo R (2014) Quercetin: a pleiotropic kinase inhibitor against cancer. Cancer Treat Res 159: 185-205

Russo M, Spagnuolo C, Tedesco I, Bilotto S, Russo GL (2012) The flavonoid quercetin in disease prevention and therapy: facts and fancies. Biochem Pharmacol 83: 6-15

Sidhu MC, Sharma T (2013) Medicinal plants from twelve families having antidiabetic activity: a review. Am J PharmTech Res 3: 36-52

Verma HN, Singh P, Chavan RM (2014) Gold nanoparticle: synthesis and characterization. Vet World 7: 72-77

Wang J, Zhou ND, Zhu ZQ, Huang JY, Li GX (2007) Detection of flavonoids and assay for their antioxidant activity based on enlargement of gold nanoparticles. Anal Bioanal Chem 388: 1199-1205 